\title{
Scattering of ${ }^{7} \mathrm{Be}$ and ${ }^{8} \mathrm{~B}$ and the astrophysical $S_{17}$ factor
}

\author{
G. Tabacaru, ${ }^{1}$ A. Azhari, ${ }^{1, *}$ J. Brinkley, ${ }^{1}$ V. Burjan, ${ }^{2}$ F. Carstoiu,${ }^{1,3}$ Changbo Fu,${ }^{1}$ C. A. Gagliardi, ${ }^{1}$ V. Kroha, ${ }^{2}$ \\ A. M. Mukhamedzhanov, ${ }^{1}$ X. Tang, ${ }^{1, \dagger}$ L. Trache, ${ }^{1}$ R. E. Tribble, ${ }^{1}$ and S. Zhou ${ }^{4}$ \\ ${ }^{1}$ Cyclotron Institute, Texas A\&M University, College Station, Texas 77843, USA \\ ${ }^{2}$ Institute of Nuclear Physics, Czech Academy of Science, Prague-Rez, Czech Republic \\ ${ }^{3}$ Institute of Physics and Nuclear Engineering H. Hulubei, Bucharest, Romania \\ ${ }^{4}$ China Institute of Atomic Energy, P.O. Box 275(46), Beijing 102 413, China
}

(Received 23 August 2005; published 28 February 2006)

\begin{abstract}
Measurements of ${ }^{7} \mathrm{Be}$ scattering at $87 \mathrm{MeV}$ on a melamine $\left(\mathrm{C}_{3} \mathrm{~N}_{6} \mathrm{H}_{6}\right)$ target and of ${ }^{8} \mathrm{~B}$ at $95 \mathrm{MeV}$ on $\mathrm{C}$ were performed. For ${ }^{7} \mathrm{Be}$ the angular range was extended over previous measurements and monitoring of the intensity of the radioactive beam was improved. The measurements provide a renormalization of the absolute cross section of existing data on the proton transfer reaction ${ }^{14} \mathrm{~N}\left({ }^{7} \mathrm{Be},{ }^{8} \mathrm{~B}\right){ }^{13} \mathrm{C}$ and lead to improved optical-model potentials used in the incoming and outgoing channels for the distorted-wave Born approximation analysis. The results yield an updated determination of the asymptotic normalization coefficient for the virtual decay ${ }^{8} \mathrm{~B} \rightarrow$ ${ }^{7} \mathrm{Be}+p$. The new value, $\mathrm{C}_{\mathrm{tot}}^{2}\left({ }^{8} \mathrm{~B}\right)=0.466 \pm 0.049 \mathrm{fm}^{-1}$, is slightly larger than, but consistent with, the previous result. This implies an astrophysical factor, $\mathrm{S}_{17}(0)=18.0 \pm 1.9 \mathrm{eV} \mathrm{b}$, for the solar neutrino-generating reaction ${ }^{7} \mathrm{Be}(p, \gamma)^{8} \mathrm{~B}$.

DOI: 10.1103/PhysRevC.73.025808

PACS number(s): 25.60.Bx, 25.60.Je, 26.65.+t
\end{abstract}

\section{INTRODUCTION}

Measurements of neutrinos produced in ${ }^{8} \mathrm{~B} \beta$ decay have played a prominent role in our new understanding of neutrino properties (see Refs. [1-5] and references therein). ${ }^{8} \mathrm{~B}$ is produced in the sun by the ${ }^{7} \mathrm{Be}(p, \gamma){ }^{8} \mathrm{~B}$ reaction. A good understanding of this reaction rate is needed in order to calculate the expected neutrino flux in the standard solar model [4]. The determination of the astrophysical factor $S_{17}$ has been the subject of an intense experimental and theoretical effort over the past decade. This work has been summarized in several recent publications [6-8].

We previously reported measurements of the asymptotic normalization coefficients (ANCs) for ${ }^{8} \mathrm{~B}$ that we made by using the proton transfer reactions ${ }^{10} \mathrm{~B}\left({ }^{7} \mathrm{Be},{ }^{8} \mathrm{~B}\right){ }^{9} \mathrm{Be}[9]$ and ${ }^{14} \mathrm{~N}\left({ }^{7} \mathrm{Be},{ }^{8} \mathrm{~B}\right){ }^{13} \mathrm{C}[10]$. We determined the ANCs, which fix the amplitude of the tail of the overlap integral of the ground-state wave function of ${ }^{8} \mathrm{~B}$ onto the two-body channel ${ }^{7} \mathrm{Be}+p$, by comparing a distorted-wave Born approximation (DWBA) calculation with the angular distribution for the transfer reaction. The DWBA calculation required opticalmodel potentials (OMPs) for both the incoming ${ }^{7} \mathrm{Be}+{ }^{14} \mathrm{~N}$ and outgoing ${ }^{8} \mathrm{~B}+{ }^{13} \mathrm{C}$ channels. Here we report a measurement of ${ }^{7} \mathrm{Be}$ elastic scattering on a melamine $\left(\mathrm{C}_{3} \mathrm{~N}_{6} \mathrm{H}_{6}\right)$ target, in which the angular range was doubled and the monitoring of the intensity of the ${ }^{7} \mathrm{Be}$ radioactive beam was improved relative to a previous measurement [10]. The extension of the angular range allowed a better determination of the optical potential in the incoming channel. Also elastic scattering of ${ }^{8} \mathrm{~B}$ on a $\mathrm{C}$ target was measured in order to check, for the first time, the OMP that was used in the outgoing channel ${ }^{8} \mathrm{~B}+{ }^{13} \mathrm{C}$.

\footnotetext{
*Present address: Alix Partners, Dallas, TX.

${ }^{\dagger}$ Present address: Argonne National Laboratory, Argonne, IL.
}

The radioactive beam production, the experiments, and the procedure for the data reduction are described. Then results are given of calculations with new optical potentials in both the incoming and the outgoing channels. The consequences of the improved secondary beam normalization are discussed, and the new DWBA calculations are compared with the ${ }^{14} \mathrm{~N}\left({ }^{7} \mathrm{Be},{ }^{8} \mathrm{~B}\right){ }^{13} \mathrm{C}$ data from Ref. [10] in order to extract a new value for the ANCs and the corresponding astrophysical factor $\mathrm{S}_{17}$.

\section{THE EXPERIMENTS}

The ${ }^{7} \mathrm{Be}$ radioactive beam was produced and separated by the Momentum Achromatic Recoil Spectrometer (MARS) [11]. The primary beam was ${ }^{7} \mathrm{Li}$ at $18.6 \mathrm{MeV} /$ nucleon delivered by the K500 superconducting cyclotron at Texas A\&M University. It bombarded a liquid-nitrogen-cooled $\mathrm{H}_{2}$ gas target at a pressure of $2 \mathrm{~atm}$, with entrance and exit windows of $12-\mu \mathrm{m}$-thick Havar. A secondary beam of ${ }^{7} \mathrm{Be}$ at $12.5 \mathrm{MeV} /$ nucleon was filtered from other reaction products by the MARS. The characteristics of the beam spot, which were measured with a 900- $\mu$ m-thick two-dimensional positionsensitive silicon detector (PSSD) placed at the MARS focal plane (the target detector), were a spot size of $2.5 \times 3.6 \mathrm{~mm}$ FWHM (horizontal $\times$ vertical) and an angular spread of $1.8^{\circ} \times 0.6^{\circ}$. The purity of the ${ }^{7} \mathrm{Be}$ beam was $99 \%$ at an average rate of $\sim 80 \mathrm{kHz}$. Alpha particles were the primary contaminant. For a detailed description of radioactive beam production with MARS, see Ref. [12].

Following beam tuning, the secondary target, a melamine foil, was moved into the beam spot. Four $5 \times 5 \mathrm{~cm}$ PSSDs were placed symmetrically around the target on an aluminum plate, as shown in Fig. 1. Detectors 1 and 2 (110 $\mu \mathrm{m}$ thick) covered a laboratory angular range from $4^{\circ}$ to $19^{\circ}$, and detectors 3 and 4 (65 $\mu \mathrm{m}$ thick) covered $16^{\circ}$ to $30^{\circ}$. 


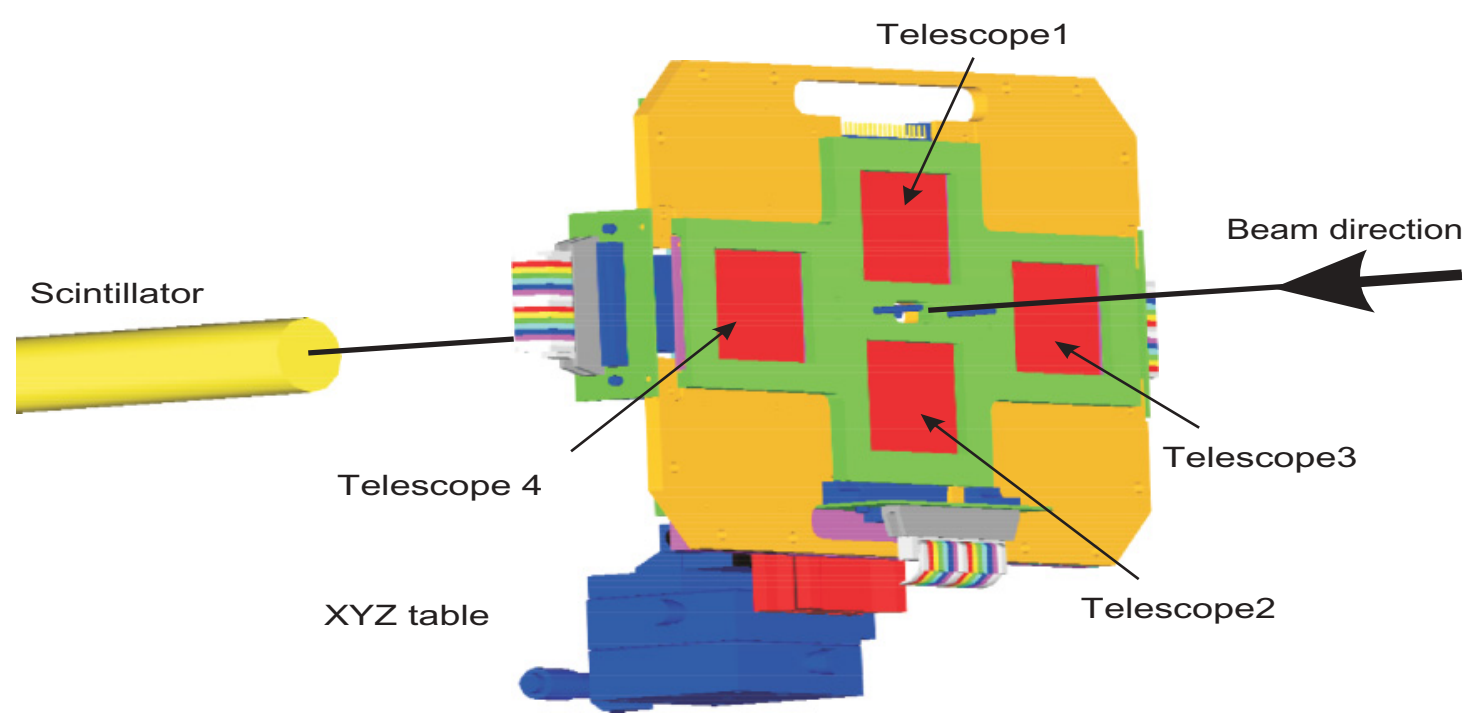

FIG. 1. (Color online) A three-dimensional view of the detector assembly.

All four PSSDs were backed by 500- $\mu$ m-thick silicon detectors providing particle identification information $(\Delta E, E)$. Each PSSD was position calibrated by use of a mask with six slots that were $0.8 \mathrm{~mm}$ wide and were spaced $8 \mathrm{~mm}$ apart. To decrease the inverse current in the detectors and minimize their noise, the detectors were cooled to approximately $-10^{\circ} \mathrm{C}$ with two electric thermocoolers fixed on the aluminum plate. The assembly was mounted on an XYZ optical table for precise positioning.

In our previous experiments with a ${ }^{7} \mathrm{Be}$ beam $[9,10]$, we determined the number of secondary beam particles indirectly by measuring the intensity of the ${ }^{7} \mathrm{Li}$ primary beam in a Faraday cup and normalizing the yield at low primary beam intensities by counting the ${ }^{7} \mathrm{Be}$ with the target detector. Periodically (typically once a day) the calibration procedure was repeated to check for any rate variations that were due to drifts in the MARS power supplies.

Following the measurements of Refs. $[9,10]$ and summarized in Ref. [12], we modified the experimental setup by adding a monitor detector to count the radioactive beam particles directly. The beam-monitoring system, which is shown in Fig. 1, used a wire-mesh screen to reduce the secondary beam intensity and a plastic scintillator coupled with a photomultiplier tube to count the radioactive beam particles that passed through the target. The old monitoring system based on a Faraday cup reading of the primary beam was run in parallel to compare the two results. In measurements with a high-intensity ${ }^{11} \mathrm{~B}$ primary beam, a difference between the two normalization procedures was observed. Beam heating reduced the density of the gas in the production target, causing a drop in the isotope production rate per nanoampere of primary beam current.

By scaling the heat deposition of the beam in the gas target, we found that this effect may have produced a small but nonnegligible shift in the beam normalization during the previous ${ }^{14} \mathrm{~N}\left({ }^{7} \mathrm{Be},{ }^{8} \mathrm{~B}\right){ }^{13} \mathrm{C}$ experiment [10]. The effect was to overestimate the number of secondary beam particles and hence reduce the extracted cross section and the resulting $\mathrm{ANC}$. In contrast, the primary beam intensity for the ${ }^{10} \mathrm{~B}\left({ }^{7} \mathrm{Be},{ }^{8} \mathrm{~B}\right){ }^{9} \mathrm{Be}$ measurement [9] was sufficiently low to have a negligible effect. For the present experiment, the monitor detector was a NE102A plastic scintillator coupled to a photomultiplier tube. To minimize rate-dependent effects in the photomultiplier tube, two screens with a transparency of $9 \%$ each were added to attenuate the beam intensity. The yield in the monitor detector was calibrated with the procedure described in Ref. [13].

For the ${ }^{8} \mathrm{~B}$ elastic-scattering measurement, the radioactive beam was produced by means of the ${ }^{1} \mathrm{H}\left({ }^{10} \mathrm{~B},{ }^{8} \mathrm{~B}\right){ }^{3} \mathrm{H}$ reaction by use of a $27-\mathrm{MeV} /$ nucleon ${ }^{10} \mathrm{~B}$ primary beam on a liquidnitrogen-cooled $\mathrm{H}_{2}$ gas target at a pressure of 3 atm, with entrance and exit windows of $50-\mu \mathrm{m}$ Havar. A $137-\mathrm{mg} / \mathrm{cm}^{2} \mathrm{Al}$ degrader was placed behind the gas cell to reduce the secondary beam energy. A 95-MeV radioactive beam of ${ }^{8} \mathrm{~B}$, with a rate of about $5 \mathrm{kHz}$, was focused at the end of the MARS. The beam purity was better than $95 \%$, with $\alpha$ particles being the primary contaminant. The full-width energy spread was limited to 1.6 MeV by momentum-defining slits. Beam emittance was optimized by a pair of slits after the last quadrupole in the MARS. The plastic scintillator behind the target was used for direct counting of the secondary beam particles, in this case without any wire-mesh screen. Two telescopes, each consisting of a $110-\mu$ m-thick PSSD backed by a $500-\mu$ m-thick $\mathrm{Si}$ detector, observed the secondary reaction products. The telescopes covered the angular range $\theta_{\mathrm{lab}}=4^{\circ}-19^{\circ}$.

In both experiments, we determined target properties such as thickness and uniformity by using the radioactive ${ }^{7} \mathrm{Be}$ and ${ }^{8} \mathrm{~B}$ beams directly by detecting beam particles at $0^{\circ}$ with and without the target. The resultant energy loss measurements were compared with calculations made with the computer code SRIM [14] to extract the thicknesses. In addition, offline measurements were made with a ${ }^{228} \mathrm{Th} \alpha$ source. The resulting target thicknesses were $1.54 \pm 0.06 \mathrm{mg} / \mathrm{cm}^{2}$ for melamine and $1.9 \pm 0.1 \mathrm{mg} / \mathrm{cm}^{2}$ for $\mathrm{C}$. 


\section{RESULTS}

\section{A. ${ }^{7}$ Be elastic scattering}

We performed the kinematic reconstruction of the elastically scattered reaction products by using the energy and position information from the four detector telescopes. The events selected corresponded to scattering of ${ }^{7} \mathrm{Be}$ on ${ }^{14} \mathrm{~N}$ and ${ }^{12} \mathrm{C}$ since the two contributions could not be separated. First, all the events with $(\Delta E, E)$ corresponding to ${ }^{7} \mathrm{Be}$ were identified. Then the correlation of ${ }^{7} \mathrm{Be}$ energy versus scattering angle was used to select those events that were consistent with elastic scattering off either ${ }^{14} \mathrm{~N}$ or ${ }^{12} \mathrm{C}$. This discriminated against scattering on $\mathrm{H}$ and inelastic scattering populating excited states in either ${ }^{14} \mathrm{~N}$ or ${ }^{12} \mathrm{C}$. However, it was not possible to separate the elastically scattered events from inelastic scattering leading to the first excited state in ${ }^{7} \mathrm{Be}$ at $E_{\mathrm{ex}}=0.429 \mathrm{MeV}$. This contribution was estimated from data obtained for inelastic scattering of ${ }^{7} \mathrm{Li}$ on ${ }^{13} \mathrm{C}$ at $63 \mathrm{MeV}$ [15] to the analog state at $E_{\mathrm{ex}}=0.477 \mathrm{MeV}$. The inelastic cross section for ${ }^{7} \mathrm{Be}$ on ${ }^{14} \mathrm{~N}$ and ${ }^{12} \mathrm{C}$ was calculated at the current energy assuming that the deformation lengths are equal for the analog states. The result was subtracted from the data. The correction was negligible at all but the largest angles, where it amounted to a few percent. The effective solid angle as a function of scattering angle was obtained from a Monte Carlo simulation that used the beam parameters and detector geometry as input [12]. Figure 2 shows the resulting angular distribution corresponding to elastically scattered ${ }^{7} \mathrm{Be}$ on ${ }^{14} \mathrm{~N}$ and ${ }^{12} \mathrm{C}$. The angular distribution predicted from the optical-model parameters used in Ref. [12] is shown as the dashed curve in Fig. 2. The Monte Carlo calculation was used to generate a smoothed DWBA angular distribution, thus taking into account the finite angular binning in the data.

The new beam normalization procedure measuring directly the secondary beam showed that when we normalized to the primary beam during the previous ${ }^{7} \mathrm{Be}+{ }^{14} \mathrm{~N}$ measurement, we

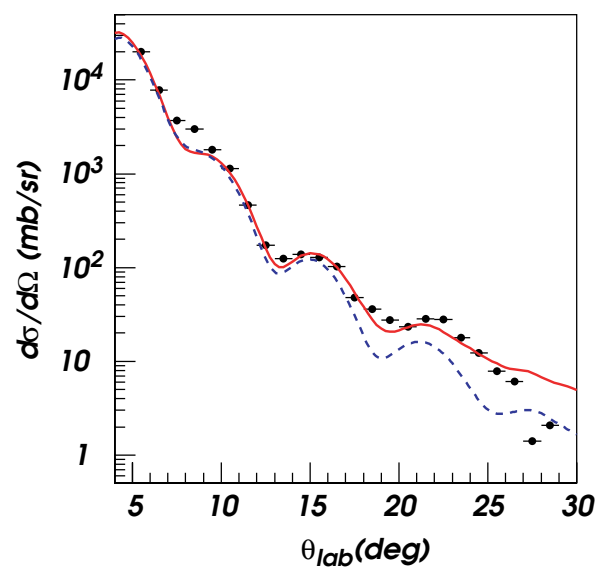

FIG. 2. (Color online) Angular distribution for elastic scattering of ${ }^{7} \mathrm{Be}$ on ${ }^{14} \mathrm{~N}$ and ${ }^{12} \mathrm{C}$. The points are experimental data after subtraction of the inelastic-scattering contribution. The solid (red) curve is the new calculation labeled E in Table I, and the dashed curve (blue) is the previous calculation from Ref. [12]. Both calculations are smeared to account for finite angular resolution. overestimated the ${ }^{7} \mathrm{Be}$ beam production by $5.5 \%$. Therefore we underestimated the cross-sections determined in Ref. [10] by this amount. This renormalization also applies to the absolute cross-section measurement for the ${ }^{14} \mathrm{~N}\left({ }^{7} \mathrm{Be},{ }^{8} \mathrm{~B}\right){ }^{13} \mathrm{C}$ reaction in Ref. [10]. The former value for the ${ }^{8} \mathrm{~B}$ ANC is scaled by a factor of 1.055 in Eq. (3).

With the renormalization, the results of the new measurement are compatible with those reported in Ref. [10] over the same angular range. However, the data at larger angles lie above the DWBA prediction, suggesting that a stronger absorption was used than is needed. To obtain a better description of the elastic scattering, calculations were carried out with a range of new parameters.

The optical parameters used in Ref. [10] were based on results from an analysis of elastic scattering of loosely bound $p$-shell nuclei [16], which demonstrated that the data can be described with double-folded potentials. The potentials quoted in Ref. [16] were obtained from calculated nuclear matter densities folded with an effective nucleon-nucleon interaction (JLM (Jeukenne-Lejeune-Mahaux) [17]), smeared (two range parameters, $t_{V}$ and $t_{W}$ ) and renormalized (two strength parameters, $N_{V}$ and $N_{W}$ ) to produce

$$
U_{\mathrm{DF}}(r)=N_{V} V\left(r, t_{V}\right)+i N_{W} W\left(r, t_{W}\right)
$$

The calculations for the previous studies were done with the JLM1 effective interaction with standard range parameters: $t_{V}=1.20 \mathrm{fm}, t_{W}=1.75 \mathrm{fm}$, and average renormalizations $N_{V}=0.37, N_{W}=1.00$ (for details see Ref. [16] and references therein). These parameters served as the starting point for the new calculations. Elastic scattering of ${ }^{7} \mathrm{Be}$ at $87.7 \mathrm{MeV}$ on ${ }^{14} \mathrm{~N}$ and ${ }^{12} \mathrm{C}$ were calculated in the center-of-mass frame, then transformed into the lab frame and added with weights 1.0 and 0.5 , respectively, equal to the ratio of ${ }^{14} \mathrm{~N}$ to ${ }^{12} \mathrm{C}$ nuclei in the melamine. The resulting curve was smoothed by use of the Monte Carlo code. The parameters for the folding potential were varied together for both target nuclei. This is justified since both ${ }^{14} \mathrm{~N}$ and ${ }^{12} \mathrm{C}$ are well bound and have similar densities in the surface region. The extended angular coverage of the present data was still not sufficient to attempt an optical-model fit with free parameters. Rather, the two normalization and two range parameters were varied. The parameters for various calculations and the reduced $\chi^{2}$ values obtained by comparison with the data are presented in Table I.

We obtained four entries in the table (A, B, C, and $\mathrm{H}$ ) by adjusting the renormalization of the real and the imaginary parts of the potential. The smearing ranges of the interaction, $t_{V}$ and $t_{W}$, were adjusted for three cases $(\mathrm{D}, \mathrm{E}$, and $\mathrm{F})$, and the density dependence was adjusted in one case $(G)$ for which the JLM2 interaction was used. The best results were obtained for cases D, E, G, and H. The small differences between the $\chi^{2}$ values show that it is difficult to choose a "best solution." Rather, DWBA calculations for the ${ }^{14} \mathrm{~N}\left({ }^{7} \mathrm{Be},{ }^{8} \mathrm{~B}\right){ }^{13} \mathrm{C}$ transfer reaction were carried out for five of the potentials, and the results are compared with the previous calculations in Table I. 
TABLE I. The optical-model parameters, the corresponding $\chi^{2}$ per degree of freedom for the ${ }^{7} \mathrm{Be}$ elastic-scattering fits, the calculated DWBA cross section for the ${ }^{14} \mathrm{~N}\left({ }^{7} \mathrm{Be},{ }^{8} \mathrm{~B}\right){ }^{13} \mathrm{C}$ reaction (see text), and the ratio of the calculated DWBA cross section to that in Ref. [10].

\begin{tabular}{|c|c|c|c|c|c|c|c|}
\hline Calculation & $N_{V}$ & $N_{W}$ & $t_{V}(\mathrm{fm})$ & $t_{W}(\mathrm{fm})$ & $\chi^{2} / N$ & $\tilde{\sigma}_{\mathrm{DW}}\left(\mathrm{mb} / \mathrm{fm}^{-1}\right)$ & $\begin{array}{c}\text { Ratio } \\
\mathcal{R}=\frac{\sigma_{\mathrm{DW}}(\text { new })}{\sigma_{\mathrm{DW}}(\text { orig })}\end{array}$ \\
\hline Ref. [10] & 0.37 & 1.00 & 1.20 & 1.75 & 35.19 & 2.469 & 1.000 \\
\hline A & 0.45 & 0.90 & 1.20 & 1.75 & 10.72 & & \\
\hline B & 0.40 & 0.92 & 1.20 & 1.75 & 15.02 & 2.385 & 0.966 \\
\hline $\mathrm{C}$ & 0.42 & 0.92 & 1.20 & 1.75 & 12.84 & & \\
\hline $\mathrm{D}$ & 0.42 & 0.90 & 0.80 & 1.75 & 10.31 & 2.408 & 0.975 \\
\hline $\mathrm{E}$ & 0.42 & 0.90 & 0.80 & 1.55 & 7.97 & 2.538 & 1.028 \\
\hline $\mathrm{F}$ & 0.52 & 0.78 & 0.12 & 2.59 & 25.72 & & \\
\hline $\mathrm{G}$ & 0.40 & 0.85 & 1.20 & 1.75 & 7.49 & 2.375 & 0.962 \\
\hline $\mathrm{H}$ & 0.40 & 0.85 & 1.20 & 1.75 & 9.22 & 2.137 & 0.900 \\
\hline
\end{tabular}

\section{B. ${ }^{8}$ B experiment}

A similar analysis was used for the ${ }^{8} \mathrm{~B}$ elastic scattering on a natural $\mathrm{C}$ target. The resulting angular distribution is shown in Fig. 3, where it is compared with calculations made with the folded potentials by use of the average parameters $t_{V}=1.20 \mathrm{fm}, t_{W}=1.75 \mathrm{fm}, N_{V}=0.37$, and $N_{W}=1.00$. The solid (dashed) curve shows the results after (before) smoothing with the Monte Carlo calculation. The ${ }^{8} \mathrm{~B}$ density used in the folding procedure was that calculated in Ref. [16] with the correct ANC for the last proton. Because of the limited angular range of the data, no attempts were made to improve the fit by adjusting parameters. Based on the similar densities for ${ }^{12} \mathrm{C}$ and ${ }^{13} \mathrm{C}$ and on results found in cases in which scattering on both ${ }^{12} \mathrm{C}$ and ${ }^{13} \mathrm{C}$ were measured, the parameters $\left(t_{V}, t_{W}\right.$, $\left.N_{V}, N_{W}\right)$ used for the natural $\mathrm{C}$ target were assumed to be valid for the ${ }^{8} \mathrm{~B}+{ }^{13} \mathrm{C}$ channel in DWBA calculations of the transfer reaction.

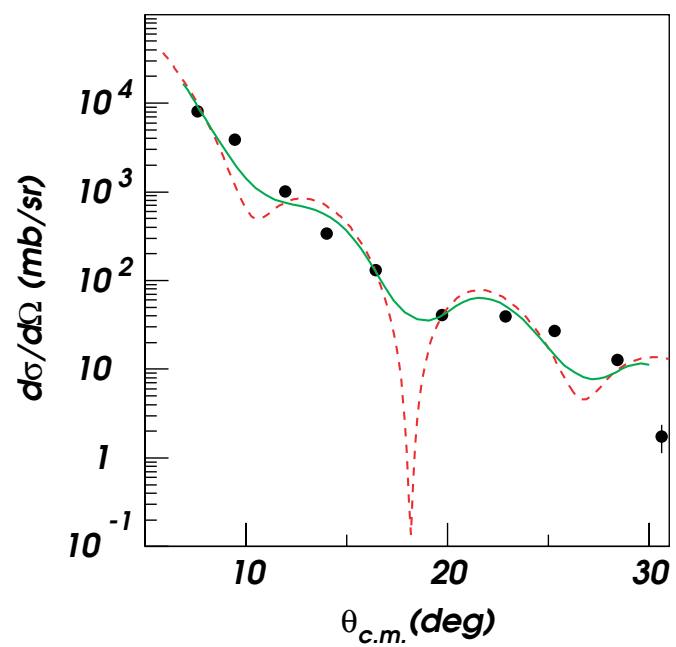

FIG. 3. (Color online) The angular distribution for elastic scattering of ${ }^{8} \mathrm{~B}$ on $\mathrm{C}$. The dashed curve is the calculated cross section, and the solid curve is the result after the finite angular resolution is accounted for.

\section{IMPLICATIONS FOR ANCs}

To extract the ANCs, four terms must be calculated to account for the possible proton transitions:

$$
\begin{aligned}
& \frac{d \sigma}{d \Omega}=\mathrm{C}_{8 \mathrm{~B}, p_{3 / 2}}^{2}\left[\frac{\mathrm{C}_{{ }^{4} \mathrm{~N}, p_{1 / 2}}^{2}}{b_{{ }^{2} \mathrm{~N}, p_{1 / 2}}^{2}} \frac{\sigma_{p_{1 / 2} \rightarrow p_{3 / 2}}}{b_{8_{\mathrm{B}, p_{3} / 2}}^{2}}+\frac{\mathrm{C}_{14}^{2} \mathrm{~N}, p_{3 / 2}}{b_{14}^{2} \mathrm{~N}, p_{3 / 2}} \frac{\sigma_{p_{3 / 2} \rightarrow p_{3 / 2}}}{b_{8_{\mathrm{B}, p_{3 / 2}}^{2}}^{2}}\right] \\
& +\mathrm{C}_{{ }^{\mathrm{B}, p_{1 / 2}}}^{2}\left[\frac{\mathrm{C}_{{ }^{14} \mathrm{~N}, p_{1 / 2}}^{2}}{b_{{ }^{2} \mathrm{~N}, p_{1 / 2}}^{2}} \frac{\sigma_{p_{1 / 2} \rightarrow p_{1 / 2}}}{b_{8_{\mathrm{B}, p_{1 / 2}}^{2}}^{2}}+\frac{\mathrm{C}_{{ }^{14} \mathrm{~N}, p_{3 / 2}}^{2}}{b_{14}^{2} \mathrm{~N}, p_{3 / 2}} \frac{\sigma_{p_{3 / 2} \rightarrow p_{1 / 2}}}{b_{8}^{2} \mathrm{~B}, p_{1 / 2}}\right] \\
& =\mathrm{C}^{2}\left({ }^{8} \mathrm{~B}, p_{3 / 2}\right)\left[\widetilde{\sigma}_{\mathrm{DW}}\right] \text {, }
\end{aligned}
$$

where the $\sigma$ 's are the calculated DWBA differential cross sections for proton transfer from the $p_{3 / 2}$ and $p_{1 / 2}$ orbitals in ${ }^{14} \mathrm{~N}$ to the $p_{3 / 2}$ and $p_{1 / 2}$ orbitals in ${ }^{8} \mathrm{~B}$, the $b_{l j}$ 's are the ANCs for the single-particle orbitals used in the DWBA calculation, and the $\mathrm{C}_{14} \mathrm{~N}, l j$ 's and $\mathrm{C}_{{ }^{8} \mathrm{~B}, l j}$ 's are the ANCs for ${ }^{14} \mathrm{~N} \rightarrow{ }^{13} \mathrm{C}+$ $p$ and ${ }^{8} \mathrm{~B} \rightarrow{ }^{7} \mathrm{Be}+p$, respectively. The ANCs, $\mathrm{C}_{{ }^{14} \mathrm{~N}, p_{1 / 2}}=$ 18.2(0.9) $\mathrm{fm}^{-1}$ and $\mathrm{C}_{14}^{2} \mathrm{~N}, p_{3 / 2}=0.91(14) \mathrm{fm}^{-1}$, were measured in Refs. $[18,19]$. Since the contributions from the two different orbitals cannot be disentangled in the transfer reaction, it is necessary to fix the ratio of the two terms. In the previous analysis, this was done by the introduction of $\delta^{2}=\mathrm{C}^{2}\left({ }^{8} \mathrm{~B}, p_{1 / 2}\right) /$ $\mathrm{C}^{2}\left({ }^{8} \mathrm{~B}, p_{3 / 2}\right)=0.157$, based on theoretical calculations. This quantity has now been measured to be $\delta^{2}=0.125(20)$ in the mirror neutron transfer reaction ${ }^{13} \mathrm{C}\left({ }^{7} \mathrm{Li},{ }^{8} \mathrm{Li}\right){ }^{12} \mathrm{C}$ [15]. The factor $\delta^{2}$ was used to rewrite Eq. (2) with a single ANC, as shown.

DWBA calculations were carried out with the code PTOLEMY [20]. The results of the calculations are given in Table I, in which the value shown in column 7 is the quantity in the last square brackets in Eq. (2), $\widetilde{\sigma}_{\mathrm{DW}}$, integrated over the angular region $\theta_{\text {c.m. }}=4^{\circ}-25^{\circ}$ to match the data. This quantity contains the DWBA cross sections weighted with the ANCs for ${ }^{14} \mathrm{~N}$, the single-particle ANCs calculated for the appropriate Woods-Saxon proton-binding potentials in ${ }^{8} \mathrm{~B}$ and ${ }^{14} \mathrm{~N}$, and the mixing ratio in the ground state of ${ }^{8} \mathrm{~B}, \delta^{2}$. Since the reaction is peripheral, the results do not depend on the geometry assumed for the proton-binding potentials, which are chosen to be of Woods-Saxon shape with depths adjusted to 
reproduce the experimental proton-binding energies in ${ }^{8} \mathrm{~B}$ and ${ }^{14} \mathrm{~N}$, respectively. The results shown were calculated with the reduced radius $r_{0}=1.20 \mathrm{fm}$, the diffuseness $a=0.60 \mathrm{fm}$, and the spin-orbit term from Ref. [21]. The exit channel parameters were fixed to the previous values as confirmed by the present ${ }^{8} \mathrm{~B}$ elastic-scattering results. Calculations were done at $E_{\text {lab }}=83.5 \mathrm{MeV}$, the energy of the previous experiment, with optical-model parameter sets $\mathrm{B}, \mathrm{D}, \mathrm{E}, \mathrm{G}$, and $\mathrm{H}$ from Table I. In column 8 the ratios of the present calculations to the same quantities calculated in Ref. [10] are given. The average of the five results, weighted by the $\chi^{2}$,s, gives the ratio $\langle\mathcal{R}\rangle=0.968 \pm 0.047$. The new ANC, calculated with the relation

$$
\mathrm{C}_{{ }^{B}, p_{3 / 2}}^{2}(\text { new })=(1.055 /\langle\mathcal{R}\rangle) \mathrm{C}_{8}^{2} \mathrm{~B}, p_{3 / 2}(\text { old }),
$$

is $C_{8 \mathrm{~B}, p_{3 / 2}}^{2}$ (new) $=0.414 \pm 0.043 \mathrm{fm}^{-1}$. The overall uncertainty contains contributions from statistics (2.6\%), absolute normalization of the cross section (5\%), input parameters in the Monte Carlo simulation of the experiment (1.4\%), and uncertainties in the ANC for the ${ }^{14} \mathrm{~N}$ vertex (4.9\%). The contribution of each of these factors remains the same as in the original analysis [12]. However, the current accuracy for the absolute value of the cross section is improved compared with that of the previous experiment because of the change in beam normalization. The uncertainty that was due to the optical-model parameters was taken from the standard deviation of the calculated cross sections (column 7 or 8 in Table I) and is $7 \%$ compared with the previous value of $8.1 \%$.

The relation between the ANCs and the astrophysical factor $\mathrm{S}_{17}(0)$, in units of electron volts times barns, is [22]

$$
\mathrm{S}_{17}(0)=38.6\left(\mathrm{C}_{p_{3 / 2}}^{2}+\mathrm{C}_{p_{1 / 2}}^{2}\right)=38.6 \mathrm{C}_{p_{3 / 2}}^{2}\left(1+\delta^{2}\right) .
$$

The new value of the ANC gives $S_{17}(0)=18.0 \pm 1.9 \mathrm{eV} b$. This value is very close to that obtained from the ${ }^{10} \mathrm{~B}\left({ }^{7} \mathrm{Be},{ }^{8} \mathrm{~B}\right){ }^{9} \mathrm{Be}$ reaction in Ref. [9], which produced $\mathrm{S}_{17}(0)=$ $18.4 \pm 2.5 \mathrm{eV} \mathrm{b}$. The weighted average of the two results is $\mathrm{S}_{17}(0)=18.2 \pm 1.8 \mathrm{eV} \mathrm{b}$.

\section{CONCLUSION}

Elastic scattering of ${ }^{7} \mathrm{Be}$ at about $12 \mathrm{MeV} /$ nucleon has been measured over an extended angular range on a melamine target.
The results provide a renomalization of previous data and a better determination of the optical-model parameters used for the entrance channel of the ${ }^{14} \mathrm{~N}\left({ }^{7} \mathrm{Be},{ }^{8} \mathrm{~B}\right){ }^{13} \mathrm{C}$ reaction. For the first time elastic scattering of ${ }^{8} \mathrm{~B}$ was measured on a $\mathrm{C}$ target, thus allowing for a check of the optical-model parameters used for the exit channel in the DWBA calculation. Also, the mixing ratio between the $1 p_{1 / 2}$ and $1 p_{3 / 2}$ components was taken from a measurement of the $\left({ }^{7} \mathrm{Li},{ }^{8} \mathrm{Li}\right)$ reaction [15], rather than a theoretical prediction. These improvements lead to the revised value of the ANC for ${ }^{8} \mathrm{~B} \rightarrow{ }^{7} \mathrm{Be}+p$ from the ${ }^{14} \mathrm{~N}\left({ }^{7} \mathrm{Be},{ }^{8} \mathrm{~B}\right){ }^{13} \mathrm{C}$ reaction of $\mathrm{C}^{2}\left({ }^{8} \mathrm{~B}, p_{3 / 2}\right.$; new $)=0.414 \pm 0.043 \mathrm{fm}^{-1}$, resulting in $\mathrm{C}_{\text {tot }}^{2}\left({ }^{8} \mathrm{~B}\right.$; new $)=\mathrm{C}_{p_{3 / 2}}^{2}+\mathrm{C}_{p_{1 / 2}}^{2}=0.466 \pm 0.049 \mathrm{fm}^{-1}$. This, in turn leads to a larger value for the astrophysical $\mathrm{S}$ factor for the ${ }^{7} \mathrm{Be}(\mathrm{p}, \gamma)^{8} \mathrm{~B}$ reaction, $\mathrm{S}_{17}(0)=18.0 \pm$ $1.9 \mathrm{eV} b$ and an average from measurements on two different targets of $\mathrm{S}_{17}(0)=18.2 \pm 1.8 \mathrm{eV} \mathrm{b}$.

Our result for $\mathrm{S}_{17}(0)$ is a bit over $2 \sigma$ lower than the extrapolation of the most recent and precise direct measurement of ${ }^{7} \mathrm{Be}(p, \gamma)^{8} \mathrm{~B}$ by Junghans et al. [7]. Our central value is about $1.5 \sigma$ lower than the average central value obtained by Cyburt et al. [8] in a recent analysis that uses all of the best available capture data, under the assumption that they are independent. Including the uncertainty quoted by Cyburt et al., our results are consistent at the $1 \sigma$ level. The reason for the discrepancy between the ANC result and the extrapolated value from Junghans et al. is not understood. However, we note that direct measurements with both radioactive beams and targets and indirect measurements continue to be carried out on this important proton capture reaction.

\section{ACKNOWLEDGMENTS}

This work was supported in part by the U.S. Department of Energy under grant no. DE-FG02-93ER40773, the U.S. National Science Foundation under grant no. INT-45952100001, the Romanian Ministry for Education, Research and Youth under contract no. 555/2000, and the Robert A. Welch Foundation. F. Carstoiu acknowledges the support of the Cyclotron Institute, Texas A\&M University, for the part of the time this work was done.
[1] R. Davis, D. Harmer, and K. C. Hoffman, Phys. Rev. Lett. 20, 1205 (1968).

[2] S. Fukuda et al. (Super-Kamiokande Collaboration), Phys. Rev. Lett. 86, 5651 (2001).

[3] Q. R. Ahmed et al. (SNO Collaboration), Phys. Rev. Lett. 87, 071301 (2001).

[4] J. N. Bahcall and M. H. Pinsonneault, Phys. Rev. Lett. 92, 121301 (2004).

[5] W. Haxton, P. Parker, and C. Rolfs, arXiv:nucl-th/0501020 (2005).

[6] B. Davids and S. Typel, Phys. Rev. C 68, 045802 (2003).

[7] A. R. Junghans, E. C. Mohrmann, K. A. Snover, T. D. Steiger, E. G. Adelberger, J. M. Casandjian, H. E. Swanson, L. Buchmann, S. H. Park et al., Phys. Rev. C 68, 065803 (2003).
[8] R. H. Cyburt, B. Davids, and B. K. Jennings, Phys. Rev. C 70, 045801 (2004).

[9] A. Azhari, V. Burjan, F. Carstoiu, H. Dejbakhsh, C. A. Gagliardi, V. Kroha, A. M. Mukhamedzhanov, L. Trache, and R. E. Tribble, Phys. Rev. Lett. 82, 3960 (1999).

[10] A. Azhari, V. Burjan, F. Carstoiu, C. A. Gagliardi, V. Kroha, A. M. Mukhamedzhanov, X. Tang, L. Trache, and R. E. Tribble, Phys. Rev. C 60, 055803 (1999).

[11] R. E. Tribble, A. Azhari, C. A. Gagliardi, J. C. Hardy, A. M. Mukhamedzhanov, X. Tang, L. Trache, and S. J. Yennello, Nucl. Phys. A701, 278C (2002).

[12] A. Azhari, V. Burjan, F. Carstoiu, C. A. Gagliardi, V. Kroha, A. M. Mukhamedzhanov, F. M. Nunes, X. Tang, L. Trache, and R. E. Tribble, Phys. Rev. C 63, 055803 (2001). 
[13] X. Tang, A. Azhari, C. A. Gagliardi, A. M. Mukhamedzhanov, F. Pirlepesov, L. Trache, R. E. Tribble, V. Burjan, V. Kroha, and F. Carstoiu, Phys. Rev. C 67, 015804 (2003).

[14] J. F. Ziegler, J. P. Biersack, and U. Littmark, The Stopping and Ranges of Ions in Matter, Vol I: The Stopping and Range of Ions in Solids (Pergamon, New York, 1985).

[15] L. Trache, A. Azhari, F. Carstoiu, H. L. Clark, C. A. Gagliardi, Y.-W. Lui, A. M. Mukhamedzhanov, X. Tang, N. Timofeyuk, and R. E. Tribble, Phys. Rev. C 67, 062801(R) (2003).

[16] L. Trache, A. Azhari, H. L. Clark, C. A. Gagliardi, Y.-W. Lui, A. M. Mukhamedzhanov, R. E. Tribble, and F. Carstoiu, Phys. Rev. C 61, 024612 (2000).

[17] J. P. Jeukenne, A. Lejeune, and C. Mahaux, Phys. Rev. C 16, 80 (1977).
[18] L. Trache, A. Azhari, H. L. Clark, C. A. Gagliardi, Y. W. Lui, A. M. Mukhamedzhanov, R. E. Tribble, and F. Carstoiu, Phys. Rev. C 58, 2715 (1998).

[19] P. Bem, V. Burjan, V. Kroha, J. Novak, S. Piskor, E. Simeckova, J. Vincour, C. A. Gagliardi, A. M. Mukhamedzhanov, and R. E. Tribble, Phys. Rev. C 62, 024320 (2000).

[20] M. Rhoades-Brown, M. H. Macfarlane, and S. C. Pieper, Phys. Rev. C 21, 2417 (1980).

[21] L. Trache, A. Kolomiets, S. Shlomo, K. Heyde, H. Dejbakhsh, C. A. Gagliardi, R. E. Tribble, X. G. Zhou, V. E. Iacob, and A. M. Oros, Phys. Rev. C 54, 2361 (1996).

[22] H. M. Xu, C. A. Gagliardi, R. E. Tribble, A. M. Mukhamedzhanov, and N. K. Timofeyuk, Phys. Rev. Lett. 73, 2027 (1994). 